\title{
Colloidal SERS sensors for inflammatory biomarker detection in urine for patient risk stratification
}

\author{
Rachel L. Kidd ${ }^{1 \dagger}$, William J. Anderson ${ }^{2}$, Josh P. Prince ${ }^{2 \dagger}$, Akosua Agyemang-Prempeh ${ }^{3 \dagger}$, Peter Roach ${ }^{2}$, \\ Tracey A. Newman ${ }^{1 *}$ and Sumeet Mahajan ${ }^{2,4^{*}}$ \\ ${ }^{1}$ Clinical \& Experimental Sciences, University of Southampton, SO16 6YD \\ ${ }^{2}$ School of Chemistry, University of Southampton, SO17 1BJ, UK \\ ${ }^{3}$ Institute for Sound and Vibration Research, University of Southampton, SO17 1BJ, UK \\ ${ }^{4}$ Institute for Life Sciences, University of Southampton, SO17 1BJ, UK
}

KEYWORDS Surface-enhanced Raman, colloidal SERS, inflammation detection, biomarker quantification, urinalysis

\begin{abstract}
Consistently raised levels of inflammatory marker - Neopterin, in urine is linked to increased risk of progression of age-related disease and poorer prognosis. We have developed colloidal SERS sensors and demonstrate their ability, and ease of use, for quantification of neopterin in human urine samples. Results with the sensors are comparable and in agreement with those obtained by HPLC and allow individuals to be stratified into 'risk' categories based on their results. With an average $17.85 \%$ difference in results between the two analytical approaches, SERS with colloidal sensors, demonstrates an alternative method that is rapid, inexpensive, requires minimal sample treatment, can be performed on a portable instrument with little need for complex data analysis, whilst having the analytical strength to reliably demonstrate an individual's risk category based on inflammatory load.
\end{abstract}

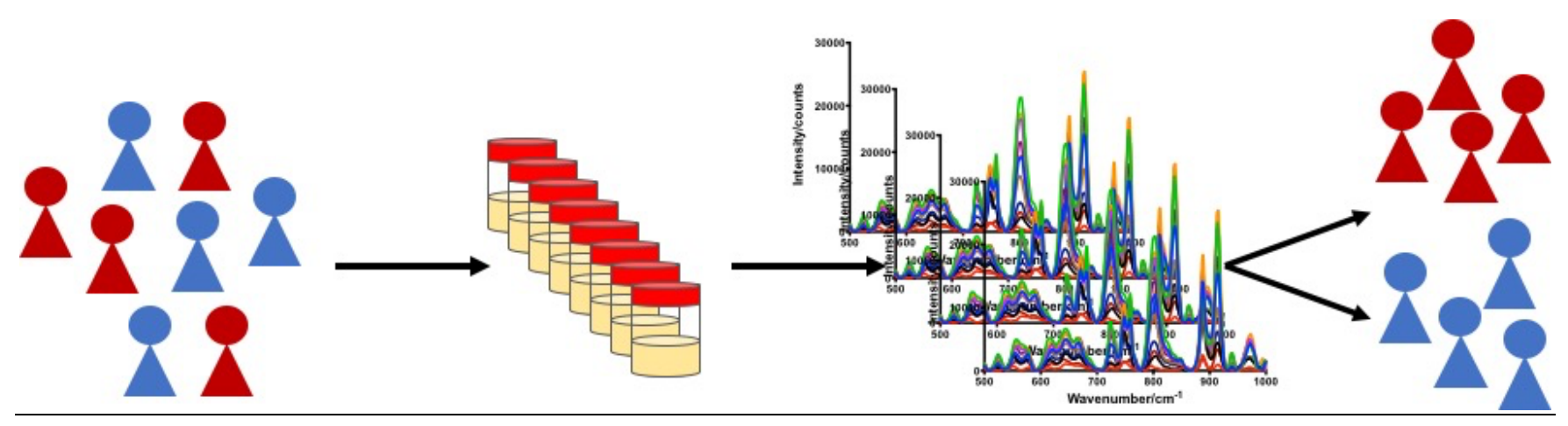

Surface-enhanced Raman spectroscopy (SERS) is a highly sensitive analytical technique with low limits of detection (down to $\mathrm{fM}$ and single-molecule detection) ${ }^{1-3}$. It utilizes the localized surface plasmon resonance (LSPR) of metallic nanomaterials to dramatically enhance Raman signals ${ }^{4-7}$. An analyte must be located in close proximity to, or adsorbed onto, the metallic nanoscale surface in order for its Raman signature to be enhanced. SERS has become increasingly employed for quantification of biologically relevant molecules ${ }^{8-15}$ as it provides molecularly specific information, is label-free and has the ability to be performed in aqueous environments such as biofluids including urine ${ }^{16-20}$. Raman spectroscopy provides several advantages over other well-known analytical methods, including the use of portable spectrometers, allowing for point-of-care analysis of clinical samples.
Reproducible SERS enhancements require strict control over the material's nanostructure and the location of the analyte. High enhancement is commonly obtained using colloidal SERS wherein nanoparticles are aggregated resulting in plasmon "hot spots". However, this approach offers poor control over the "hot spots" and spatial proximity of the analyte to them resulting in low measurement reproducibility ${ }^{21}$.

One way to improve quantification is to use the Standard addition method (SAM) which is a well-established technique in analytical chemistry. SAM is useful when dealing with complex matrices, where many analytes may compete for the "hot spot", preventing accurate quantification. SAM works by spiking known amounts of the analyte of interest into the sample, a plot of peak area against concentration of spiked analyte will enable the unknown amount of analyte to be calculated. Several groups have demonstrated the use of SAM to quantify analytes in urine 
$11,22,23$, however the extra steps of spiking samples with multiple known amounts of target analyte, adds additional time and expense in terms of number of samples, number of measurements required, and having a stock of target analyte. Therefore, such a method is less suitable for point-of-care SERS measurements. Another way to improve reproducibility is to employ an internal standard as first reported by Bell et al. who used an internal standard to compensate for temporal signal variation when quantifying dipicolinic acid from Bacillus spores ${ }^{24,}$. . One problem with internal standards is competitive adsorption between target analyte and standard that may occur at the metal surface and the method will not work above saturation of the nanoparticle surface ${ }^{26}$ and therefore the ratio of analyte to standard may not be a linear relationship. However, placing the internal standard molecules within the inside of core-shell nanoparticles has been shown to improve the quantitative analysis of target molecules over a large concentration range ${ }^{27}$. Use of spacer molecules to control the nanogap between colloidal nanoparticles has also been used to control and improve reproducibility ${ }^{28}$ however, this still requires use of aggregation agents which may interfere with the analytes in solution.

An alternative approach is to use layer-by-layer (LbL) selfassembly to "freeze" plasmonic nanogaps between nanoparticles on a planar or colloidal substrate. Fabrication of plasmontunable, sensitive, quantitative and self-calibrating colloidal SERS sensors have been reported by our group earlier ${ }^{29}$. Alternating coatings of gold nanoparticles (AuNPs) and polyelectrolyte "spacer" layers controls the nanoparticle spacing in the radial direction. AuNPs are adsorbed directly from their colloidal suspension without addition of any salt. Consequently, AuNP assembly creates a uniform deposition without polydisperse aggregates, overall enabling the controlled fabrication of reproducible AuNP sensors which have been shown to be sensitive and reproducible quantitative sensors.

Neopterin is a small biomolecule present in urine which can be used as a marker of systemic inflammation. Systemic inflammation has been shown to play a role in health prognosis in a multitude of age-related conditions such as frailty ${ }^{30}$, cardiovascular disease $\mathrm{e}^{31}$, hearing loss $\mathrm{s}^{32,33}$ and dementia ${ }^{34}$. Neopterin is produced from guanosine triphosphate by human monocytes and macrophages after stimulation by interferon gamma (IFN$\gamma$ ) derived from antigen-activated T lymphocytes ${ }^{35}$. Compared to blood, urine sampling is non-invasive. Urine is also less protein-rich, so is less likely to suffer from matrix effects. Urine carries abundant metabolic information which can reflect health conditions within the body. Neopterin is expressed as urinary neopterin-to-creatinine ratio (UNCR, measured in $\mu \mathrm{MM}^{-1}$ ) to normalize for glomerular filtration rate. A healthy non-inflammed individual will have a UNCR $<251 \mu \mathrm{MM}^{-135-38}$. The main techniques used for the analysis of neopterin in urine include enzyme-linked immunosorbent assay (ELISA) ${ }^{39}$, radioimmunoassay (RIA) ${ }^{38}$ and high performance liquid chromatography (HPLC) $)^{37,38}$. ELISA and RIA lack sensitivity and require costly reagents, HPLC is considered the gold standard technique, however this is a lab-based method, time-consuming and expensive; requiring complex equipment and highly trained personnel. As an alternative Raman spectroscopy offers portable instrumentation capable of point and shoot analysis, potentially enabling the technique to be used in a point-of-care situation by clinical staff.

Label-free SERS analysis of urine has been largely confined to studies of cancers ${ }^{40-43}$, diseases ${ }^{44,45}$ and drugs ${ }^{13,46}$. Rather than absolute quantification of a biomarker, most of these studies rely on multivariate analysis to classify a sample as healthy or diseased. For example, Huang et al. ${ }^{41}$ applied silver nanoparticles to the urine of esophageal cancer patients and healthy volunteers, then used principal component analysis (PCA) combined with linear discriminant analysis (LDA) to differentiate the SERS spectra between normal and cancerous urine samples. Their results demonstrate diagnostic sensitivity of $87.5 \%$ and specificity of $83.3 \%$. Label-free SERS analysis of urine without multivariate analysis has been demonstrated ${ }^{11,46-49}$. Westley et al. ${ }^{11}$ have demonstrated absolute quantification of uric acid in urine without the need for multivariate analysis by employing the SAM. Their method shows good agreement against HPLC with an average of $<9 \%$ difference between the analytical approaches. However, despite improving quantitative accuracy, the SAM requires extra sample preparation and increased sample analysis, rendering it unsuitable for rapid point-of-care testing.

In this work we have developed a method that uses SERS sensors for quantitative analysis of an inflammatory marker (neopterin) in urine. Raman measurement of neopterin in biofluids has been demonstrated ${ }^{14,50-52}$, but to our knowledge this is the first example of neopterin quantification in urine. We show for the first time that unlike other SERS substrates, our sensors can be used as a colloidal suspension, simply mixing with a urine sample for analysis. We compare the SERS performance of our sensors with colloidal nanoparticles and show huge improvement in reproducibility and storage stability. Our quantification results with expected values and that obtained through the established HPLC 'gold standard' confirm the utility of our method. The method has the benefit of requiring no sample pretreatment or multivariate analysis, thus making it suitable for point-of-care testing with a portable spectrometer. This technique offers high sensitivity, specificity, simplicity and cost-effectiveness. Such an analytical system available at point-of-care will allow long-term monitoring of patient's immune system activation which can be revolutionary in monitoring of disease and health prognosis.

\section{Experimental /Materials and Methods}

\section{Sensor fabrication}

SERS sensors were fabricated using a LbL method described by Anderson et al. ${ }^{29}$ However, here Silica microparticles (7.5 $\mu \mathrm{M}$, Microparticles $\mathrm{GmbH}$ Berlin) were used as a core, instead of polystyrene, on which iterative layers of Poly(ethyleneimine) (PEI) $\left(M_{w} \sim 750000 \mathrm{~g} \mathrm{M}^{-1} 50 \%\right.$ (wt) in water, SigmaAldrich), poly(sodium 4-styrenesulfonate) (PSS) ( $M_{w} \sim 70000$ $\mathrm{g} \mathrm{M}^{-1}$, Sigma-Aldrich) and citrate-capped AuNPs (40nm, $8 \%$ $\mathrm{CV}, 9 \times 10^{10}$ particles per $\mathrm{ml}$, BBI Solutions) were applied to form two AuNP layers. Sensors were diluted and stored in water at $4^{\circ} \mathrm{C}$ until use.

\section{Urine Preparation}

Synthetic urine was prepared according to the method of Villa and Poppi ${ }^{53}$. Sodium chloride ( $\mathrm{NaCl}$, Acros organics), potassium chloride (KCl, Sigma-Aldrich), sodium dihydrogen phosphate $\left(\mathrm{NaH}_{2} \mathrm{PO}_{4}\right.$, Sigma-Aldrich), urea (Sigma-Aldrich), citric acid (Fisher) and bovine albumin (Fisher) were added to $18.2 \Omega$ Millipore water and $\mathrm{pH}$ adjusted with sodium hydroxide (NaOH, Sigma-Aldrich). Synthetic urine was stored at $4^{\circ} \mathrm{C}$ until use. 
Human urine samples were collected from healthy adults aged 65-75 years (NRES REC: 13/SC/0507 and University of Southampton ERGO 7923). When received, the urine samples were centrifuged at $2000 \mathrm{rpm}$ at a temperature of $4^{\circ} \mathrm{C}$ for 10 minutes and stored at $-20^{\circ} \mathrm{C}$. Prior to use samples were thawed at $4^{\circ} \mathrm{C}$ and kept in the dark with a foil covering, as neopterin is highly light sensitive. Prior to HPLC analysis, both synthetic and human urine samples were treated to remove protein by using spin-columns (Vivaspin $500 \mu \mathrm{L} 5 \mathrm{kD}$, Sigma-Aldrich) and the filtrate taken for HPLC analysis.

\section{HPLC analysis}

HPLC separation was conducted using a Gilson HPLC system (with a Shimadzu RF-10A Fluorescence detector and a Gilson UV/Vis- 155 detector) set up for reverse-phase using a 150 x $4.6 \mathrm{~mm}$ column with a $5 \mu \mathrm{m}$ particle size (Gemini C18 110A). For each injection, the run time was 40 minutes. The mobile phase consisted of $25 \mathrm{mM}$ Acetic Acid, 10mM Sodium 1-octane sulfonate at $\mathrm{pH} 3$. The organic buffer with $50 \%$ acetonitrile was increased in gradient from $0 \%$ to $100 \%$ over 30 minutes at a flow rate of $1.0 \mathrm{~mL} \mathrm{~min}^{-1}$. The addition of the sodium-1-octane sulfonate (Sigma-Aldrich) acts as an ion-pairing reagent to increase retention time. Urine samples were analysed both undiluted and at a 1:4 dilution. Each sample $(20 \mu \mathrm{l})$ was introduced using an autoinjector. The retention time for creatinine was 21 minutes and neopterin 10 minutes although these retention times shifted earlier when the human urine samples were analysed. Neopterin was detected by fluorescence (excitation $353 \mathrm{~nm}$, emission $438 \mathrm{~nm}$ ) and creatinine detected by UV absorbance $(235 \mathrm{~nm})$. Repeated measurements were made for each sample and averaged. For creatinine the spectra were background subtracted for water. Spectral analysis was performed in UniPoint software.

\section{SERS analysis}

SERS analysis was performed on a Renishaw InVia spectrometer (633nm HeNe laser, 6mW max output). The instrument was calibrated using a silicon wafer with a static spectrum peak at $521 \mathrm{~cm}^{-1}$. Lbl SERS sensors $(0.1 \%, 5 \mu \mathrm{L})$ were mixed with analyte/sample $(20 \mu \mathrm{L})$ by pipetting and measurements taken after a 10 -minute incubation. A drop $(5 \mu \mathrm{L})$ of mixture was then pipetted onto an aluminium disc and the microscope used to focus onto a single sensor. A spectral acquisition consisted of a single scan $\left(500-1000 \mathrm{~cm}^{-1}\right.$ for neopterin measurement and $1500-2000 \mathrm{~cm}^{-1}$ for creatinine measurement (although for some initial experiments a creatinine peak was used in the $500-1000 \mathrm{~cm}^{-1}$ range)) with an exposure time of 10 seconds using a long working distance objective (Olympus, 50x, 0.55 N.A.). For each sample between 6 and 10 spectra were collected. Processing was performed with iRootlab ${ }^{54}$ and Renishaw's WiRE software. Spectra were denoised using wavelet smoothing and baseline corrected with an $8^{\text {th }}$ order polynomial in iRootlab. WiRE was used to curve fit and determine peak areas. For the batch reproducibility measurements, the measured creatinine peak $\left(685 \mathrm{~cm}^{-1}\right)$ was not normalised to another. For the other results, the peak area of the peaks at $1760 \mathrm{~cm}^{-1}$ and $1850 \mathrm{~cm}^{-1}$ were taken with the $1760 \mathrm{~cm}^{-1}$ peak being from creatinine and the $1850 \mathrm{~cm}^{-1}$ peak being from the polyelectrolyte within the sensors and therefore acting as an internal standard to normalize the creatinine peak to. Similarly, for neopterin, the peak area of the peak at $695 \mathrm{~cm}^{-1}$ was normalized to another internal standard peak at $887 \mathrm{~cm}^{-1}$. The wavenumber regions of the extended scans were chosen to focus on known peaks for neopterin and creatinine.

\section{Data Analysis}

All data analysis was performed in GraphPad Prism 8 including correlation, Bland-Altman plots, paired t-tests and descriptive statistics. Mean peak areas were interpolated from standard curves produced for both neopterin and creatinine with both HPLC and SERS.

\section{Results and Discussion}

\section{SERS sensor optimization}

The Lbl SERS sensors have been fabricated to achieve controlled spacing between the two layers of gold nanoparticles. Two polyelectrolytes are used to form these spacing layers. Figure 1 shows a schematic of a fully assembled SERS sensor, showing the layers and the formation of a "hot spot" between the two layers of gold nanoparticles. The fabrication process enables tight control over the separation of nanoparticles in the radial direction, therefore controls the formation of "hot spots" with some degree of precision. In comparison to using colloidal gold nanoaprticles for SERS enhancement, these SERS sensors should provide improved reproducibility.

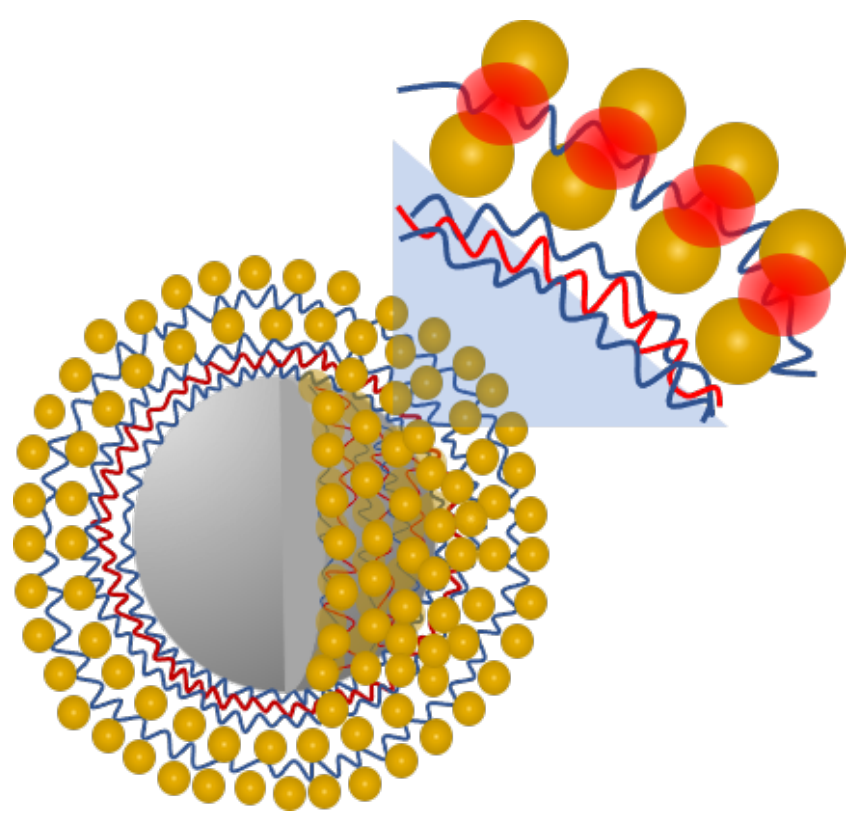

Figure 1. Schematic of SERS sensors. Sensors were fabricated using a LBL process of coating a silica microparticle with polyelectrolyte and two layers of AuNPs. The spacer layer defines the separation between the nanoparticle layers in the radial direction and thus "hot spot" formation between the two layers, producing reproducible SERS sensors.

UV-Vis scattering has been used to demonstrate the evolution of LSPR in the colloidal SERS sensors (Figure $2 a$ ). The $\lambda_{\max }$ at $590 \mathrm{~nm}$ represents singular AuNPs, whereas the red shifted $\lambda_{\max }$ at $850 \mathrm{~nm}$ represents coupled AuNPs within the two layers on the SERS sensors. This information confirms that the two layers of AuNPs are spaced in close enough proximity for LSPR to couple and form "hotspots". This is similar to the $\lambda_{\max }$ shift seen when colloidal AuNPs are aggregated, however, in the case of SERS sensors the coupling is controlled as the AuNPs are fixed 
in space. Previous work has shown that colloidal SERS sensors prepared with 2 layers of AuNPs optimizes the SERS signal
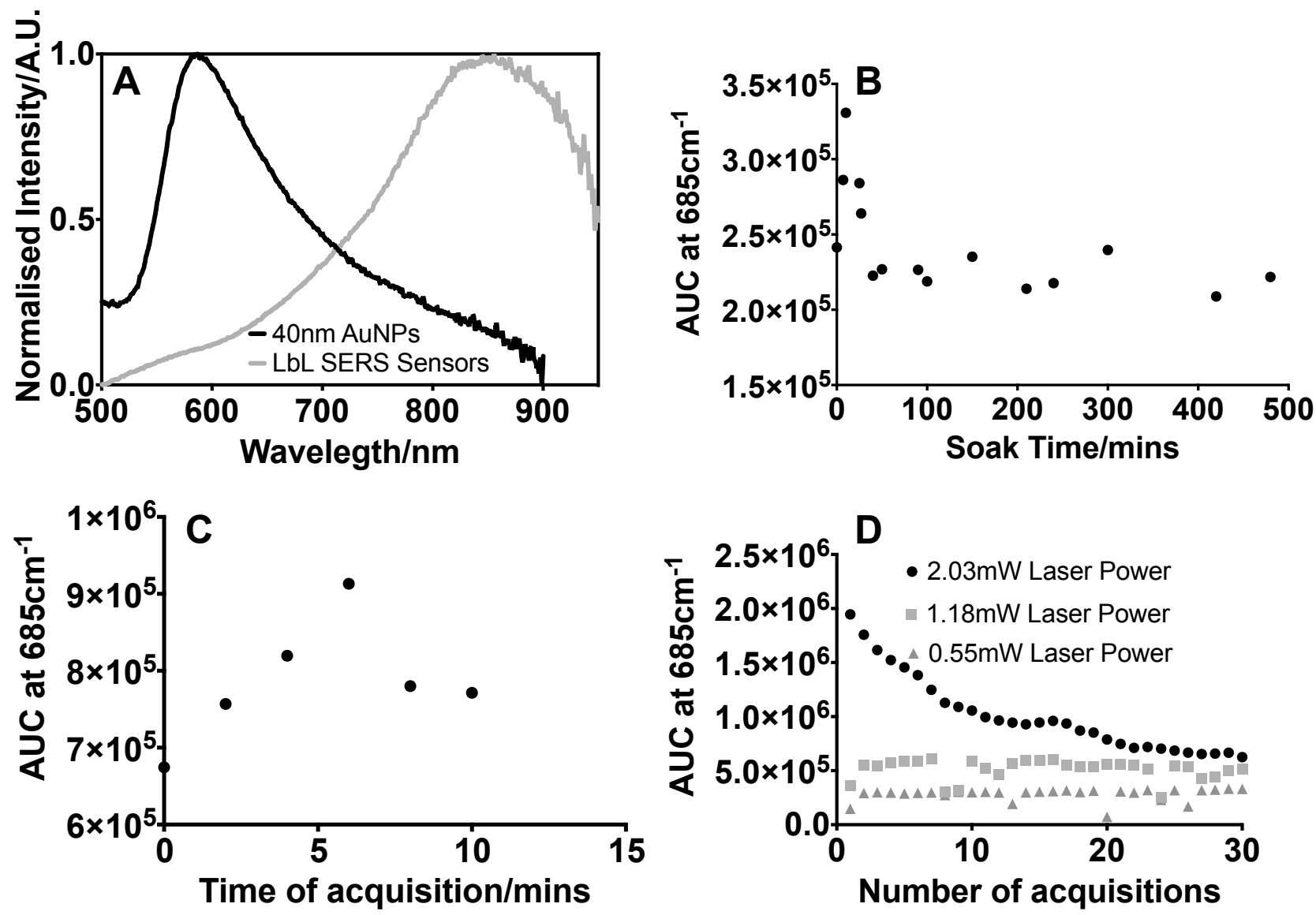

Figure 2. (a) UV-Vis scattering measurement of prepared SERS sensors and colloidal 40nm AuNPs demonstrating evolution of LSPR. (b) Area under curve of creatinine peak with increasing soak time of analyte with sensor. An increase and subsequent decrease in intensity is observed, with optimum soak time being 10-20 minutes. (c) Area under curve of creatinine peak with time of acquisition. After 6 minutes (4 acquisitions) intensity obtained decreases as sensor degrades. (d) Area under curve of creatinine peak when multiple spectra are taken using a single sensor. With 100\% laser power, maximum intensity is obtained however intensity decreases with each acquisition as sensor degrades.

achieved using a $633 \mathrm{~nm}$ excitation source ${ }^{29}$. In order to establish the optimum conditions for using the SERS sensors a number of experiments were performed. Figure $2 \mathrm{~b}$ shows that after a 10-minute soak of the analyte/sample with the colloidal sensors, the SERS intensity obtained is at a peak, which then starts to decrease. For this reason, we chose to incubate every sample with colloidal SERS sensors for 10 minutes prior to analysis. When multiple spectra are acquired from a single SERS sensor, the signal and therefore sensor are seen to deteriorate (figure $2 \mathrm{c}$ and $2 \mathrm{~d}$ ). Reducing laser power helps alleviate this, however also leads to a reduction in the signal-tonoise which was not conducive to achieve the highest sensitivities possible for detection and quantitation. Hence, for quantitative analysis we have used maximum laser power but each SERS acquisition is made from a separate SERS sensor within the sample rather than taking multiple acquisitions from a single sensor.

\section{SERS sensor batch reproducibility and stability}

Assembly of the colloidal SERS sensors using the Lbl process was standardized to be as consistent as possible to ensure the SERS enhancement properties of the sensors would be uniform. The reliability and stability of the fabrication process was assessed by taking multiple (100) SERS spectra of a standard sample (5mM creatinine) with two different batches of freshly prepared SERS sensors, a batch that had been stored at $4{ }^{\circ} \mathrm{C}$ for one month, and with salt-aggregated $40 \mathrm{~nm}$ colloidal AuNPs. All the spectra were analysed, and area under the curve of the peak at $675 \mathrm{~cm}^{-1}$ plotted. Figure 3 shows the distribution of values within each batch as well as the consistently improved enhancement achieved with SERS sensors over 40nm colloidal AuNPs. The mean areas under the peak for batch $\mathrm{A}$ and $\mathrm{B}$ were $7.0 \times 10^{5}$ and $7.1 \times 10^{5}$ respectively, compared to a mean of $4.1 \times 10^{4}$ when using the colloidal AuNPs. The variance within batch A was $26.0 \%$ (RSD) and batch B $27.8 \%$ (RSD) compared to a variance of $120.3 \%$ (RSD) between the 100 spectra taken with the colloidal AuNPs. Similarly, the batch-to-batch reproducibility was evaluated by performing a one-way ANOVA. No significant difference $(p=0.9655)$ was shown between the measurements from batch $\mathrm{A}$ and $\mathrm{B}$, however a significant difference $(\mathrm{p}<$ 0.0001 ) was observed when batch $A$ and $B$ were compared to 40nm colloidal AuNPs. The stability of the SERS sensors is also demonstrated with a one-way ANOVA, showing no 
significant difference $(\mathrm{p}<0.0001)$ between the measurements taken with freshly prepared SERS sensors and those that had been stored for one month at $4^{\circ} \mathrm{C}$.

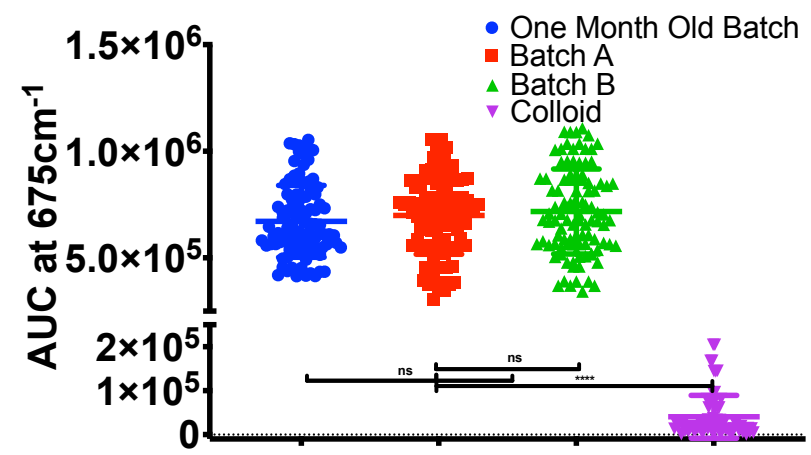

Figure 3. Verification of batch-to-batch reproducibility of SERS sensors and improved enhancement over colloidal AuNPs. Mean (RSD) values: One-month old batch $6.7 \times 10^{5}$ (25.1), Batch A $7.0 \times 10^{5}$ (26.0), Batch B $7.1 \times 10^{5}$ (27.8), Colloid $4.1 \times 10^{4}(120.3)$.

These results demonstrate the increased enhancement of the SERS spectrum observed with the SERS sensors compared to SERS spectra obtained using salt-aggregated colloidal AuNPs. The within-batch reproducibility of the spectra obtained with SERS sensors shows variation (RSD 26.0 and $27.8 \%$ ) but with significant improvement over the reproducibility obtained with colloidal AuNPs (RSD 120.3\%). Similar sample preparation and measurement is required whether using the SERS sensors or the colloidal AuNPs; therefore, the improved reproducibility with no additional sample preparation is a method enhancement. Analysis of the batch-to-batch reproducibility of the SERS sensors shows no difference in results obtained, suggesting there is no variation in formation or uniformity of the sensor in the manufacturing process. The SERS sensors do not deteriorate on storage which is a huge advantage for field and point-of-care applications.

\section{Validation with synthetic urine samples}

The use of colloidal SERS sensors similar to that used here has been demonstrated previously and optimized conditions established ${ }^{29}$. However, this is the first use of such silica-core based sensors in a complex matrix such as urine. A concern in the analysis of urine is being able to detect the analytes of interest accurately in a matrix of competing molecules and signals. We aimed to establish whether physiologically relevant concentrations of creatinine and neopterin could be detected and quantified and how the obtained measurements compared to both expected values and that obtained via HPLC measurement. Synthetic urine samples were prepared with known concentrations of creatinine and neopterin and blinded prior to analyses. The concentrations calculated from the SERS measurement were found to be in very good agreement with the expected concentration of each sample ( $\mathrm{R}^{2}$ of 0.961 and 0.991 for creatinine and neopterin) as shown in figure 4. Additionally, the agreement between the two analytical methods was good ( $\mathrm{R}^{2}$ of 0.936 and 0.990 for creatinine and neopterin), demonstrating that at physiologically relevant concentrations, both methods perform equally well. Measurements of neopterin have a larger associated error (error bars are $95 \%$ confidence intervals) which is most likely due to the much lower concentrations of the samples and possible deterioration of neopterin due to its light sensitivity ${ }^{38}$.

Measurement of clinically relevant biomarker in urine

To further evaluate the validity of the colloidal SERS sensors, experiments were repeated with human urine samples from a cohort of volunteers with unknown amounts of creatinine and neopterin. As we were unaware of the concentrations within the samples, and the likely high concentrations of creatinine which risked saturating the system, it was deemed appropriate to measure each sample undiluted and as a 1:4 dilution so that both analytes could be measured in an optimum concentration range based on the concentration profiles already established for both HPLC and SERS.

The characteristic peaks for neopterin and creatinine were at $695 \mathrm{~cm}^{-1}$, and $1760 \mathrm{~cm}^{-1}$ and these were both normalized to peaks present in the colloidal SERS sensors from the polyelectrolyte layers acting as an internal standard. Having an inbuilt internal standard within the SERS sensor allows normalization of the analyte peaks to that of the standard, to take into account any variations in intensity.

Table 1 shows a summary of the results from the two analytical approaches for each analyte, along with their associated percentage difference and the corresponding UNCR. As an example, for sample 03510, there was no difference in the creatinine predicted concentration between HPLC and SERS, HPLC predicted a neopterin concentration of $1.45 \mu \mathrm{M}$, compared to $1.24 \mu \mathrm{M}$ predicted from SERS, a difference of $15.61 \%$. Overall, for all samples analysed, the percentage difference between the two analytical approaches ranged from $0 \%$ to $40 \%$, with the average being $12.77 \%$ for creatinine and $17.85 \%$ for neopterin. Although somewhat larger than ideal, this is still encouraging considering the low concentrations being measured (for neopterin) and the simplicity, ease and speed of the method compared to others published, demonstrating the real potential for point-of-care testing.

To establish the reproducibility of this SERS approach, we have analysed 6 samples (one synthetic and 5 human urine) in triplicate (figure 5). Batch-to-batch variation and reproducibility of SERS enhancement are highly important factors for determining a SERS substrate's suitability for quantitative measurement ${ }^{55}$. Inconsistent enhancements are often seen with solution based substrates due to variations in colloidal concentrations and nanoparticle aggregation. Our colloidal SERS sensors overcome these issues and we have already shown that there is no statistical significance between measurements taken with separate batches (figure 3). 
A

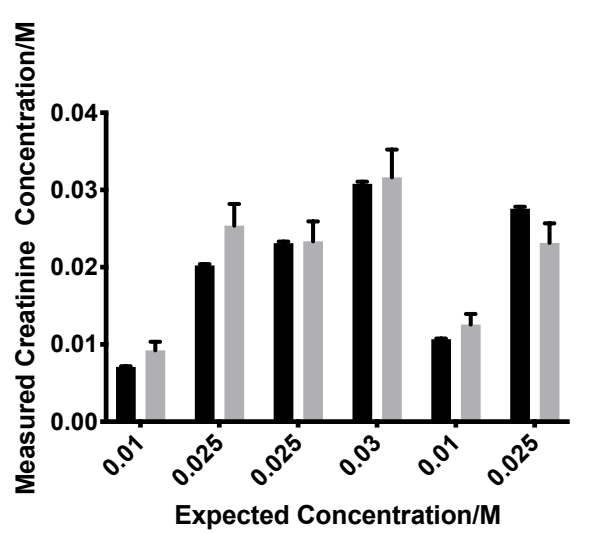

B

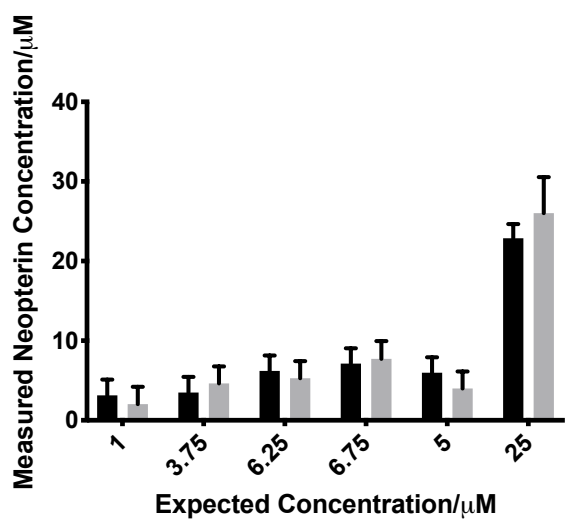

C

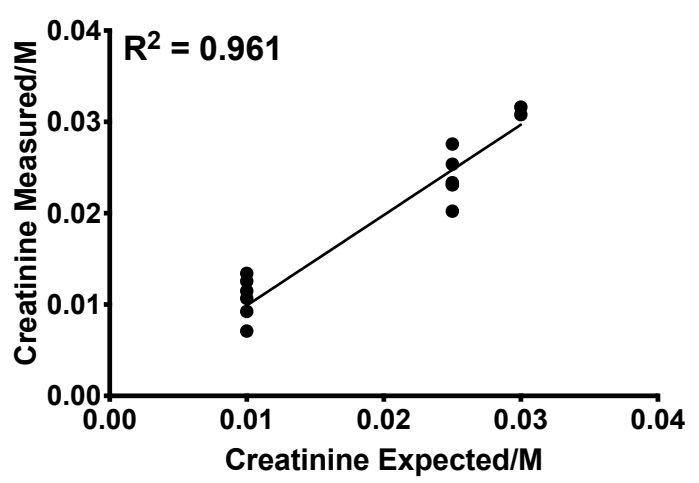

D

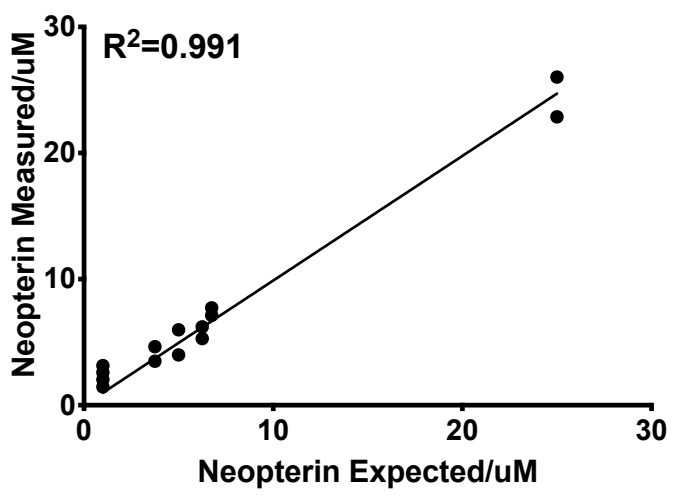

Figure 4. Results of expected vs measured synthetic urine samples. A and B) Expected vs measured concentrations of prepared synthetic urine samples analysed by SERS with SERS sensors and HPLC. Good agreement between SERS measurement and HPLC is observed. Error bars demonstrate 95\% confidence intervals. C and D) Correlation between expected and measured concentration (for SERS) is $\mathrm{R}^{2}=0.961$ and $\mathrm{R}^{2}=0.991$ for creatinine and neopterin respectively.

Table 1. Summary of results for human urine samples: HPLC, SERS and the associated percentage difference (RSD) between the two analytical approaches for neopterin $(\mu \mathrm{M})$, creatinine(M) and $\mathrm{UNCR}\left(\mu \mathrm{MM}^{-1}\right)$.

\begin{tabular}{|l|l|l|l|l|l|l|l|l|l|}
\hline Sample & $\begin{array}{l}\text { Creatinine } \\
\text { HPLC/M }\end{array}$ & $\begin{array}{l}\text { Creatinine } \\
\text { SERS/M }\end{array}$ & $\begin{array}{l}\text { Differ- } \\
\text { ence/\% }\end{array}$ & $\begin{array}{l}\text { Ne- } \\
\text { opterin } \\
\text { HPLC } \mu \mathrm{M}\end{array}$ & $\begin{array}{l}\text { Ne- } \\
\text { opterin } \\
\text { SERS/ } \mu \mathrm{M}\end{array}$ & $\begin{array}{l}\text { Differ- } \\
\text { ence/\% }\end{array}$ & $\begin{array}{l}\text { UNCR } \\
\text { HPLC/ } \mu \mathrm{MM}- \\
1\end{array}$ & $\begin{array}{l}\text { UNCR } \\
\text { SERS/ } \mu \mathrm{MM}- \\
1\end{array}$ & $\begin{array}{l}\text { Differ- } \\
\text { ence/\% }\end{array}$ \\
\hline $\begin{array}{l}\text { SAMPLE 1 } \\
\text { (0577) }\end{array}$ & 0.006 & 0.006 & 0.000 & 0.70 & 0.71 & 1.42 & 121.13 & 126.93 \\
\hline $\begin{array}{l}\text { SAMPLE 2 } \\
\text { (03510) }\end{array}$ & 0.004 & 0.004 & 0.000 & 1.45 & 1.24 & 15.61 & 340.30 & 296.05 \\
\hline $\begin{array}{l}\text { SAMPLE 3 } \\
\text { (0549) }\end{array}$ & 0.004 & 0.003 & 28.571 & 0.81 & 0.99 & 20.00 & 225.98 & 295.53 \\
\hline $\begin{array}{l}\text { SAMPLE 4 } \\
\text { (05712) }\end{array}$ & 0.002 & 0.002 & 0.000 & 0.77 & 0.52 & 38.76 & 368.46 & 26.67 \\
\hline
\end{tabular}




\begin{tabular}{|l|l|l|l|l|l|l|l|l|l|}
\hline $\begin{array}{l}\text { SAMPLE 5 } \\
\text { (05711) }\end{array}$ & 0.010 & 0.009 & 10.526 & 2.50 & 3.40 & 30.51 & 257.89 & 391.16 & 41.07 \\
\hline $\begin{array}{l}\text { SAMPLE 6 } \\
\text { (0207) }\end{array}$ & 0.010 & 0.009 & 10.526 & 3.79 & 3.44 & 9.68 & 381.31 & 363.72 & 4.72 \\
\hline $\begin{array}{l}\text { SAMPLE 7 } \\
\text { (05412) }\end{array}$ & 0.015 & 0.012 & 22.222 & 3.58 & 3.47 & 3.12 & 238.47 & 288.80 & 19.09 \\
\hline $\begin{array}{l}\text { SAMPLE 8 } \\
\text { (0518) }\end{array}$ & 0.008 & 0.006 & 28.571 & 2.13 & 2.63 & 21.01 & 264.83 & 410.76 & 43.20 \\
\hline $\begin{array}{l}\text { SAMPLE 9 } \\
\text { (0309) }\end{array}$ & 0.006 & 0.004 & 40.000 & 1.22 & 0.82 & 39.22 & 196.93 & 215.90 & 9.19 \\
\hline $\begin{array}{l}\text { SAMPLE 10 } \\
\text { (04810) }\end{array}$ & 0.016 & 0.016 & 0.000 & 4.22 & 3.56 & 16.97 & 263.78 & 229.39 & 13.95 \\
\hline $\begin{array}{l}\text { SAMPLE 11 } \\
\text { (0458) }\end{array}$ & 0.013 & 0.017 & 26.67 & 6.49 & 6.34 & 2.34 & 512.47 & 374.55 & 31.10 \\
\hline
\end{tabular}

As an example, for sample 0577 , the percentage difference in the three measurements obtained for creatinine was 3.98\% and for neopterin $15.11 \%$. The average percentage difference for triplicate analysis is $12.45 \%$ for creatinine and $17.75 \%$ for neopterin, thus demonstrating reasonable precision and overall reproducibility.

As a quick and simple analytical alternative to HPLC that doesn't require any sample preparation steps (other than dilution and mixing), addition of standards such as that required for SAM, use of expensive immunochemistry or multivariate analysis; quantification with colloidal SERS sensors can be achieved with good enough accuracy and precision to be considered a valid method.

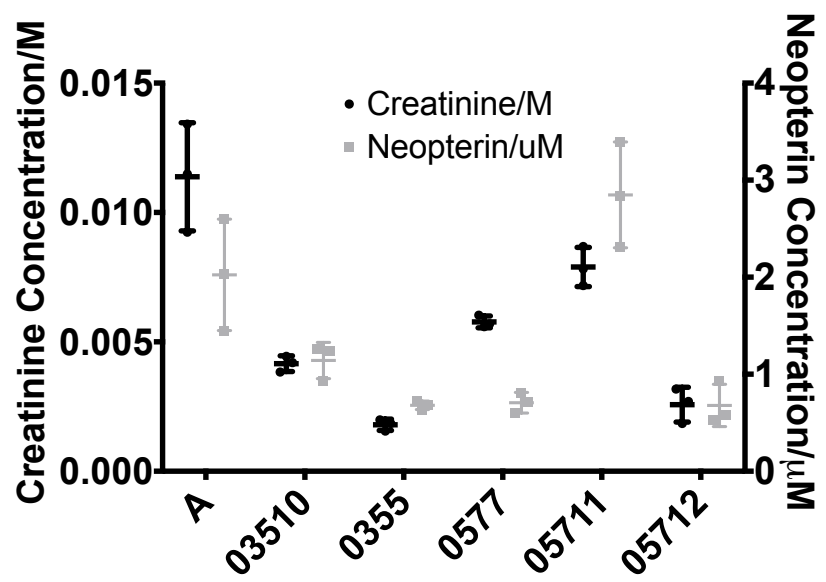

Figure 5 Summary of results for triplicate analysis of samples. Average concentration from triplicate measurement for neopterin $(\mu M)$, creatinine(M) and the relative standard deviation (RSD, \% difference) between measurements.
Stratification of results into 'risk' categories

We have demonstrated that SERS using the colloidal sensors can quantify two analytes of interest, creatinine and neopterin, in the complex matrix of urine. The benefit of measuring these two compounds is to determine the urinary neopterin-to-creatinine ratio (UNCR) as a marker of immune system activation. Individuals with a consistently raised UNCR $\left(>251 \mu \mathrm{MM}^{-1}\right)$ are more susceptible to age-related diseases and poorer prognosis of disease. Therefore, can this analytical method be used to determine a clinically relevant factor? i.e. which individuals are 'at risk' of poorer disease prognosis. After calculating the UNCR for each urine sample, we have then stratified it as high or low risk depending on the $251 \mu \mathrm{MM}^{-1}$ threshold and compared the stratification results to those obtained via the HPLC measurements and looked at the agreement (taking the HPLC measurements as gold standard).

Assuming the stratification based on HPLC results to be accurate (as gold standard), we found 8 of our 11 samples made the correct assignment of high/low risk based on UNCR $<$ or $>251 \mu \mathrm{MM}^{-1}$. Two of the three samples that weren't correctly assigned are close in concentration to the $251 \mu \mathrm{mol} / \mathrm{mol}$ cutoff, therefore, it may be advisable to have a testing protocol whereby if a sample concentration comes out as within $15 \%$ of the cut-off value $(213-289 \mu \mathrm{mol} / \mathrm{mol})$, then it should be reanalysed. Nevertheless, this corresponds to a $72 \%$ accuracy and $83 \%$ sensitivity which given a small size $(\mathrm{n}=11)$ of clinical samples is to be expected. Nevertheless, this demonstrates the promise of our SERS method as an analytical tool to stratify individuals into risk categories based on their level of inflammatory marker -neopterin in their urine. 
Table 2. UNCR $\left(\mu \mathrm{MM}^{-1}\right)$. values obtained for urine samples via HPLC and SERS and agreement of risk stratification between two methods (presuming HPLC correct as gold standard). Results demonstrate agreement in risk stratification as high/low UNCR (above or below $251 \mu \mathrm{MM}^{-1}$ ) for 8 of the 11 samples, demonstrating $72 \%$ accuracy, $83 \%$ sensitivity and positive predictive value of $71 \%$.

\begin{tabular}{|l|l|l|l|}
\hline Sample & UNCR HPLC/ $\mu M^{-1}$ & UNCR SERS/ $\mu M^{-1} \mathbf{M}^{-1}$ & $\begin{array}{l}\text { Correct stratification as } \\
\text { high/low risk }\end{array}$ \\
\hline $\mathbf{0 5 7 7}$ & 121.13 & 126.93 & Yes \\
\hline $\mathbf{0 3 5 1 0}$ & 340.30 & 296.05 & Yes \\
\hline $\mathbf{0 5 4 9}$ & 225.98 & 295.53 & No (false +ve) \\
\hline $\mathbf{0 5 7 1 2}$ & 368.46 & 284.19 & Yes \\
\hline $\mathbf{0 5 7 1 1}$ & 257.89 & 391.16 & Yes \\
\hline $\mathbf{0 2 0 7}$ & 381.31 & 363.72 & Yes \\
\hline $\mathbf{0 5 4 1 2}$ & 238.47 & 288.80 & No (false +ve) \\
\hline $\mathbf{0 5 1 8}$ & 264.83 & 410.76 & Yes \\
\hline $\mathbf{0 3 0 9}$ & 196.93 & 215.90 & Yes \\
\hline $\mathbf{0 4 8 1 0}$ & 263.78 & 229.39 & No (false -ve) \\
\hline $\mathbf{0 4 5 8}$ & 512.47 & 374.55 & Yes \\
\hline
\end{tabular}

\section{Conclusions}

We have demonstrated for the first time the use of colloidal SERS sensors as a substrate for SERS of urine. The optimized conditions for using the sensors have been established and used to measure two analytes- neopterin and creatinine, with improved enhancement and reproducibility over using colloidal AuNPs and with the added advantage of storage stability.

To validate the use of colloidal SERS sensors, we analysed synthetic urine that was prepared with known concentrations of neopterin and creatinine, and established that the SERS method could quantify the concentration of each analyte with good accuracy, and there was good agreement between the SERS and HPLC results that were used for benchmarking.

We have further demonstrated the analytical capability of this technique when using human urine samples and found promising results with good reproducibility when performed in triplicate.

We can conclude that the technique provides promise as an analytical method for absolute quantification of two biologically relevant biomarkers in urine. As a tool for stratifying individuals into 'risk' categories based on their level of immune system activation, the technique works well.

The benefits of SERS as an analytical technique are clear, acquisition is fast allowing multiple spectra to be taken (averaging would improve results), portable equipment would allow it to be used at point-of-care in a clinical or home setting, it is highly sensitive and molecularly specific, and requires no need for markers or labels. Additionally, our technique requires no sample preparation or complex spectral analysis; therefore, could easily be carried out by a "untrained' individual such as clinical staff or patient.

\section{AUTHOR INFORMATION}

\section{Corresponding Author}

*Sumeet Mahajan (s.mahajan@soton.ac.uk)

*Tracey Newman (t.a.newman@soton.ac.uk

\section{Present Addresses}

$\dagger$ Department of Audiology, University Hospitals Southampton NHS Trust, SO14 0YG

$\dagger$ MRC London Institute of Medical Sciences

$\dagger$ Komfo Anokye Teaching Hospital, ENT Unit, PO Box 1934, Kumasi

\section{Author Contributions}

SM and TN equally conceptualized and supervised the project. RK carried out the work including analysis. WA contributed to the sensor preparation method. JP and PR contributed to the HPLC analysis experimental design and acquisition. A A-P undertook all urine sample collection and initial sample preparation. RK, TN and SM designed the experiments. RK wrote the first draft of the manuscript.TN and SM equally contributed to the manuscript All authors contributed and have given approval to the final version of the manuscript.

\section{ACKNOWLEDGMENT}

RK was co-funded by a studentship from the University of Southampton (Vice-Chancellor's award) awarded to TN and SM and a European Research Council Grant to SM (NanoChemBioVision 638258). Funding support from EPSRC grant (EP/T020997/1) is also acknowledged.

\section{REFERENCES}


1. Kneipp, K.; Wang, Y.; Kneipp, H.; Perelman, L. T.; Itzkan, I.; Dasari, R. R.; Feld, M. S., Single molecule detection using surface-enhanced Raman scattering (SERS). Physical review letters 1997, 78 (9), 1667

2. Nie, S.; Emory, S. R., Probing single molecules and single nanoparticles by surface-enhanced Raman scattering. science $\mathbf{1 9 9 7}$, 275 (5303), 1102-1106.

3. Yang, S.; Dai, X.; Stogin, B. B.; Wong, T.-S., Ultrasensitive surface-enhanced Raman scattering detection in common fluids. Proceedings of the National Academy of Sciences 2016, 113 (2), 268-273.

4. $\quad$ Stiles, P. L.; Dieringer, J. A.; Shah, N. C.; Duyne, R. P. V., Surface-Enhanced Raman Spectroscopy. Annual Review of Analytical Chemistry 2008, 1 (1), 601-626.

5. Jeanmaire, D. L.; Van Duyne, R. P., Surface raman spectroelectrochemistry: Part I. Heterocyclic, aromatic, and aliphatic amines adsorbed on the anodized silver electrode. Journal of Electroanalytical Chemistry and Interfacial Electrochemistry 1977, 84 (1), 1-20.

6. Albrecht, M. G.; Creighton, J. A., Anomalously intense Raman spectra of pyridine at a silver electrode. J. Am. Chem. Soc 1977, 99 (15), 5215-5217.

7. Willets, K. A.; Duyne, R. P. V., Localized Surface Plasmon Resonance Spectroscopy and Sensing. Annual Review of Physical Chemistry 2007, 58 (1), 267-297.

8. Ma, K.; Yuen, J. M.; Shah, N. C.; Walsh, J. T.; Glucksberg, M. R.; Van Duyne, R. P., In Vivo, Transcutaneous Glucose Sensing Using Surface-Enhanced Spatially Offset Raman Spectroscopy: Multiple Rats, Improved Hypoglycemic Accuracy, Low Incident Power, and Continuous Monitoring for Greater than 17 Days. Analytical Chemistry 2011, 83 (23), 9146-9152.

9. Zhang, D.; Haputhanthri, R.; Ansar, S. M.; Vangala, K.; De Silva, H. I.; Sygula, A.; Saebo, S.; Pittman, C. U., Ultrasensitive detection of malondialdehyde with surface-enhanced Raman spectroscopy. Analytical and Bioanalytical Chemistry 2010, 398 (7), 3193-3201.

10. Zhang, K.; Liu, Y.; Wang, Y.; Zhang, R.; Liu, J.; Wei, J.; Qian, H.; Qian, K.; Chen, R.; Liu, B., Quantitative SERS Detection of Dopamine in Cerebrospinal Fluid by Dual-RecognitionInduced Hot Spot Generation. ACS Applied Materials \& Interfaces 2018, 10 (18), 15388-15394.

11. Westley, C.; Xu, Y.; Thilaganathan, B.; Carnell, A. J.; Turner, N. J.; Goodacre, R., Absolute Quantification of Uric Acid in Human Urine Using Surface Enhanced Raman Scattering with the Standard Addition Method. Analytical Chemistry 2017, 89 (4), 2472 2477.

12. Wu, X.; Chen, X.; Gao, F.; Ma, W.; Xu, L.; Kuang, H.; Li, A.; Xu, C., SERS encoded nanoparticle heterodimers for the ultrasensitive detection of folic acid. Biosensors \& Bioelectronics 2016, 75, 55-58.

13. Subaihi, A.; Almanqur, L.; Muhamadali, H.; AlMasoud, N.; Ellis, D. I.; Trivedi, D. K.; Hollywood, K. A.; Xu, Y.; Goodacre, R., Rapid, Accurate, and Quantitative Detection of Propranolol in Multiple Human Biofluids via Surface-Enhanced Raman Scattering. Analytical Chemistry 2016, 88 (22), 10884-10892.

14. Kamińska, A.; Witkowska, E.; Kowalska, A.; Skoczyńska, A.; Gawryszewska, I.; Guziewicz, E.; Snigurenko, D.; Waluk, J., Highly efficient SERS-based detection of cerebrospinal fluid neopterin as a diagnostic marker of bacterial infection. Analytical and Bioanalytical Chemistry 2016, 408 (16), 4319-4327.

15. Zhao, L.; Blackburn, J.; Brosseau, C. L., Quantitative Detection of Uric Acid by Electrochemical-Surface Enhanced Raman Spectroscopy Using a Multi layered Au/Ag Substrate. Analytical Chemistry 2015, 87 (1), 441-447.

16. Ma, L.; Zhang, Z.; Li, X., Non-invasive disease diagnosis using surface-enhanced Raman spectroscopy of urine and saliva. Applied Spectroscopy Reviews 2019, 1-23.

17. Leal, L. B.; Nogueira, M. S.; Canevari, R. A.; Carvalho, L. F. C. S., Vibration spectroscopy and body biofluids: Literature review for clinical applications. Photodiagnosis and Photodynamic Therapy 2018, 24, 237-244.

18. Baker, M. J.; Hussain, S. R.; Lovergne, L.; Untereiner, V.; Hughes, C.; Lukaszewski, R. A.; Thiefin, G.; Sockalingum, G. D., Developing and understanding biofluid vibrational spectroscopy: a critical review. Chemical Society Reviews 2016, 45 (7), 1803-1818.

19. Goodacre, R.; Graham, D.; Faulds, K., Recent developments in quantitative SERS: Moving towards absolute quantification. TrAC Trends in Analytical Chemistry 2018, 102, 359 368.

20. Bonifacio, A.; Cervo, S.; Sergo, V., Label-free surfaceenhanced Raman spectroscopy of biofluids: fundamental aspects and diagnostic applications. Analytical and Bioanalytical Chemistry 2015, 407 (27), 8265-8277.

21. Shiohara, A.; Wang, Y.; Liz-Marzán, L. M., Recent approaches toward creation of hot spots for SERS detection. Journal of Photochemistry and Photobiology C: Photochemistry Reviews 2014, 21, 2-25.

22. Hidi, I. J.; Jahn, M.; Weber, K.; Bocklitz, T.; Pletz, M. W.; Cialla-May, D.; Popp, J., Lab-on-a-Chip-Surface Enhanced Raman Scattering Combined with the Standard Addition Method: Toward the Quantification of Nitroxoline in Spiked Human Urine Samples. Analytical Chemistry 2016, 88 (18), 9173-9180.

23. Mamian-Lopez, M. B.; Poppi, R. J., Standard addition method applied to the urinary quantification of nicotine in the presence of cotinine and anabasine using surface enhanced Raman spectroscopy and multivariate curve resolution. Analytica Chimica Acta 2013, 760, 53-59.

24. Bell, S. E. J.; Mackle, J. N.; Sirimuthu, N. M. S Quantitative surface-enhanced Raman spectroscopy of dipicolinic acid - towards rapid anthrax endospore detection. Analyst 2005, 130 (4), 545-549

25. Cheung, M.; Lee, W. W. Y.; Cowcher, D. P.; Goodacre, R.; Bell, S. E. J., SERS of meso-droplets supported on superhydrophobic wires allows exquisitely sensitive detection of dipicolinic acid, an anthrax biomarker, considerably below the infective dose. Chemical Communications 2016, 52 (64), 9925-9928.

26. Stewart, A.; Zheng, S.; McCourt, M. R.; Bell, S. E. J., Controlling Assembly of Mixed Thiol Monolayers on Silver Nanoparticles to Tune Their Surface Properties. ACS Nano 2012, 6 (5), 3718-3726

27. Shen, W.; Lin, X.; Jiang, C.; Li, C.; Lin, H.; Huang, J.; Wang, S.; Liu, G.; Yan, X.; Zhong, Q.; Ren, B., Reliable Quantitative SERS Analysis Facilitated by Core-Shell Nanoparticles with Embedded Internal Standards. Angewandte Chemie International Edition 2015, 54 (25), 7308-7312.

28. Taylor, R. W.; Lee, T.-C.; Scherman, O. A.; Esteban, R.; Aizpurua, J.; Huang, F. M.; Baumberg, J. J.; Mahajan, S., Precise subnanometer plasmonic junctions for SERS within gold nanoparticle assemblies using cucurbit [n] uril "glue". ACS nano 2011, 5 (5), 3878-3887.

29. Anderson, W. J.; Nowinska, K.; Hutter, T.; Mahajan, S.; Fischlechner, M., Tuning plasmons layer-by-layer for quantitative colloidal sensing with surface-enhanced Raman spectroscopy. Nanoscale 2018, 10 (15), 7138-7146.

30. Gale, C. R.; Baylis, D.; Cooper, C.; Sayer, A. A., Inflammatory markers and incident frailty in men and women: the English Longitudinal Study of Ageing. Age 2013, 35 (6), 2493-2501.

31. Christodoulidis, G.; Vittorio, T. J.; Fudim, M.; Lerakis, S.; Kosmas, C. E., Inflammation in coronary artery disease. Cardiology in review 2014, 22 (6), 279-288.

32. Verschuur, C.; Agyemang-Prempeh, A.; Newman, T. A., Inflammation is associated with a worsening of presbycusis: Evidence from the MRC national study of hearing. International Journal of Audiology 2014, 53 (7), 469-475.

33. Nash, S. D.; Cruickshanks, K. J.; Zhan, W.; Tsai, M. Y.; Klein, R.; Chappell, R.; Nieto, F. J.; Klein, B. E. K.; Schubert, C. R.; Dalton, D. S.; Tweed, T. S., Long-term Assessment of Systemic Inflammation and the Cumulative Incidence of Age-related Hearing Impairment in the Epidemiology of Hearing Loss Study. Journals of 
Gerontology Series a-Biological Sciences and Medical Sciences 2014, 69 (2), 207-214.

34. Leung, R.; Proitsi, P.; Simmons, A.; Lunnon, K.; Güntert, A.; Kronenberg, D.; Pritchard, M.; Tsolaki, M.; Mecocci, P.; Kloszewska, I., Inflammatory proteins in plasma are associated with severity of Alzheimer's disease. PloS one 2013, 8 (6), e64971.

35. Murr, C.; Widner, B.; Wirleitner, B.; Fuchs, D., Neopterin as a marker for immune system activation. Current Drug Metabolism 2002, 3 (2), 175-187.

36. Fuchs, D.; Weiss, G.; Reibnegger, G.; Wachter, H., The role of neopterin as a monitor of cellular immune activation in transplantation, inflammatory, infectious, and malignant diseases. Critical reviews in clinical laboratory sciences 1992, 29 (3-4), 307344.

37. Hausen, A.; Fuchs, D.; König, K.; Wachter, H., Determination of neopterine in human urine by reversed-phase highperformance liquid chromatography. Journal of Chromatography B: Biomedical Sciences and Applications 1982, 227 (1), 61-70.

38. Werner, E. R.; Bichler, A.; Daxenbichler, G.; Fuchs, D.; Fuith, L. C.; Hausen, A.; Hetzel, H.; Reibnegger, G.; Wachter, H., DETERMINATION OF NEOPTERIN IN SERUM AND URINE. Clinical Chemistry 1987, 33 (1), 62-66.

39. Ozmeric, N.; Baydar, T.; Bodur, A.; Engin, A. B.; Uraz, A.; Eren, K.; Sahin, G., Level of neopterin, a marker of immune cell activation in gingival crevicular fluid, saliva, and urine in patients with aggressive periodontitis. Journal of Periodontology 2002, 73 (7), 720-725.

40. Yang, T.; Guo, X.; Wu, Y.; Wang, H.; Fu, S.; Wen, Y.; Yang, H., Facile and Label-Free Detection of Lung Cancer Biomarker in Urine by Magnetically Assisted Surface-Enhanced Raman Scattering. Acs Applied Materials \& Interfaces 2014, 6 (23), 20985-20993.

41. Huang, S.; Wang, L.; Chen, W.; Feng, S.; Lin, J.; Huang, Z.; Chen, G.; Li, B.; Chen, R., Potential of non-invasive esophagus cancer detection based on urine surface-enhanced Raman spectroscopy. Laser Physics Letters 2014, 11 (11).

42. Del Mistro, G.; Cervo, S.; Mansutti, E.; Spizzo, R.; Colombatti, A.; Belmonte, P.; Zucconelli, R.; Steffan, A.; Sergo, V.; Bonifacio, A., Surface-enhanced Raman spectroscopy of urine for prostate cancer detection: a preliminary study. Analytical and Bioanalytical Chemistry 2015, 407 (12), 3271-3275.

43. Xiang, Y.; Yang, H.; Guo, X.; Wu, Y.; Ying, Y.; Wen, Y.; Yang, H., Surface enhanced Raman detection of the colon cancer biomarker cytidine by using magnetized nanoparticles of the type $\mathrm{Fe}$ 3 O 4/Au/Ag. Microchimica Acta 2018, 185 (3), 195.

44. Yang, H.; Zhao, C.; Li, R.; Shen, C.; Cai, X.; Sun, L.; Luo, C.; Yin, Y., Noninvasive and prospective diagnosis of coronary heart disease with urine using surface-enhanced Raman spectroscopy. Analyst 2018, 143 (10), 2235-2242.

45. Zou, Y.; Huang, M.; Wang, K.; Song, B.; Wang, Y.; Chen, J.; Liu, X.; Li, X.; Lin, L.; Huang, G., Urine surface-enhanced Raman spectroscopy for non-invasive diabetic detection based on a portable Raman spectrometer. Laser Physics Letters 2016, 13 (6), 065604 .

46. Meng, J.; Tang, X.; Zhou, B.; Xie, Q.; Yang, L., Designing of ordered two-dimensional gold nanoparticles film for cocaine detection in human urine using surface-enhanced Raman spectroscopy. Talanta 2017, 164, 693-699.

47. Han, Z.; Liu, H.; Meng, J.; Yang, L.; Liu, J.; Liu, J., Portable Kit for Identification and Detection of Drugs in Human Urine Using Surface-Enhanced Raman Spectroscopy. Analytical Chemistry 2015, 87 (18), 9500-9506.

48. Alharbi, O.; Xu, Y.; Goodacre, R., Detection and quantification of the opioid tramadol in urine using surface enhanced Raman scattering. Analyst 2015, 140 (17), 5965-5970.

49. Alula, M. T.; Yang, J., Photochemical decoration of magnetic composites with silver nanostructures for determination of creatinine in urine by surface-enhanced Raman spectroscopy. Talanta 2014, 130, 55-62.

50. Elumalai, B.; Prakasarao, A.; Ganesan, B.; Dornadula, K.; Ganesan, S., Raman spectroscopic characterization of urine of normal and oral cancer subjects. Journal of Raman Spectroscopy 2015, 46 (1), 84-93.

51. Kamińska, A.; Szymborski, T.; Jaroch, T.; Zmysłowski, A.; Szterk, A., Gold-capped silicon for ultrasensitive SERSbiosensing: Towards human biofluids analysis. Materials Science and Engineering: $C$ 2018, 84, 208-217.

52. Kaminska, A.; Kowalska, A. A.; Snigurenko, D.; Guziewicz, E.; Lewinski, J.; Waluk, J., ZnO oxide films for ultrasensitive, rapid, and label-free detection of neopterin by surfaceenhanced Raman spectroscopy. Analyst 2015, 140 (15), 5090-8.

53. Villa, J. E. L.; Poppi, R. J., A portable SERS method for the determination of uric acid using a paper-based substrate and multivariate curve resolution. Analyst 2016, 141 (6), 1966-1972.

54. Trevisan, J.; Angelov, P. P.; Scott, A. D.; Carmichael, P. L.; Martin, F. L., IRootLab: a free and open-source MATLAB toolbox for vibrational biospectroscopy data analysis. Bioinformatics 2013, 29 (8), 1095-1097.

55. Prochazka, M., Surface-Enhanced Raman Spectroscopy: Bioanalytical, Biomolecular and Medical Applications. In SurfaceEnhanced Raman Spectroscopy, 1 ed.; Springer International Publishing: 2016; pp 61-91. 